\title{
Plasma cytokines IL-6, IL-8, and IL-10 are associated with the development of acute respiratory distress syndrome in patients with severe traumatic brain injury
}

Imo P. Aisiku ${ }^{1 *}$, Jose-Miguel Yamal' ${ }^{2}$ Pratik Doshi ${ }^{3}$, Julia S. Benoit ${ }^{4}$, Shankar Gopinath ${ }^{5}$, Jerry C. Goodman ${ }^{6}$ and Claudia S. Robertson ${ }^{5}$

\begin{abstract}
Background: Patients with severe traumatic brain injury (TBI) are at risk of the development of acute respiratory distress syndrome (ARDS). TBI and ARDS pathophysiologic mechanisms are known to independently involve significant inflammatory responses. The literature on the association between plasma inflammatory cytokines and ARDS in patients with TBI is sparse.

Methods: The study was a secondary analysis of the safety of a randomized trial of erythropoietin and transfusion threshold in patients with severe TBI. Inflammatory markers within the first 24 hours after injury were compared in patients who developed ARDS and patients without ARDS, using Cox proportional hazards models.

Results: There were 200 patients enrolled in the study. The majority of plasma and cerebrospinal fluid (CSF) cytokine levels were obtained within 6 hours. Plasma proinflammatory markers IL-6 and IL-8 and anti-inflammatory marker IL-10 were associated with the development of ARDS (adjusted hazard ratio $(H R)=1.55$, confidence interval $(C I)=1.14,2.11$, $P=0.005$ for $\mathrm{IL}-6$; adjusted $H R=1.32, \mathrm{Cl}=1.10,1.59, P=0.003$ for $\mathrm{IL}-8)$.
\end{abstract}

Conclusion: Plasma markers of IL-6, IL-8, and IL-10 are associated with ARDS in patients with severe TBI.

Trial registration: NCT00313716 registered 4/2006

Keywords: TBI, ARDS, ALI, Cytokines, Plasma, CSF

\section{Background}

Severe head injury is associated with an early inflammatory response of which proinflammatory and antiinflammatory cytokines are critical mediators of neuroinflammation [1-4]. The extent of brain injury is determined by the severity of the primary mechanical injury and the consequences of the secondary biomolecular injury patterns [1-8]. Proinflammatory markers but not anti-inflammatory markers have been found to be upregulated in serum from patients with TBI [9]. Some inflammatory markers such as IL-6 have been reported to be a possible predictor of elevated intracranial pressure (ICP)

\footnotetext{
* Correspondence: iaisiku@partners.org

${ }^{1}$ Department of Emergency Medicine, Brigham and Women's Hospital, 75

Francis Street, Boston, MA 02115, USA

Full list of author information is available at the end of the article
}

in patients with isolated TBI [10]. These reports support an inflammatory component to the pathophysiology of severe TBI.

Acute respiratory distress syndrome (ARDS) is known to involve an intense proinflammatory and anti-inflammatory response in the lungs as demonstrated in numerous studies [11-14]. Decreased IL-10 in bronchoalveolar lavage fluid has been associated with mortality in patients with ARDS [15]. Although there is a clear relationship between inflammatory mediators and ARDS, elevated plasma cytokines have not been shown to be associated with ARDS in at-risk medical patients $[16,17]$. None of these studies included patients with TBI.

Patients with severe TBI have high mortality and ARDS is recognized as one of the significant contributors to inpatient mortality and prolonged mechanical ventilation. 
Secondary injury may involve the development of systemic inflammatory response syndrome (SIRS), ARDS, multiple organ dysfunction syndrome, or sepsis [18-20]. Recognizing that TBI and ARDS involve significant inflammatory mediators and patients with severe TBI are at risk of ARDS, we hypothesized that early proinflammatory and anti-inflammatory cytokines may be associated with the development of ARDS.

\section{Methods}

\section{Primary hypothesis and outcomes}

The objective of this study was to determine the association between cytokine levels just after severe traumatic brain injury and the risk of ARDS. The Berlin definition of ARDS events was used [21], based on acute onset within 7 days, bilateral pulmonary infiltrates consistent with pulmonary edema, impaired oxygenation (partial pressure of oxygen $(\mathrm{PaO} 2) /$ inspired oxygen fraction $(\mathrm{FiO} 2)$ ratio $<300 \mathrm{~mm}$ $\mathrm{Hg}$ ), and impaired oxygenation not fully explained by cardiac failure [21].

\section{Study design}

This is a secondary analysis of the ARDS safety outcome from the erythropoietin randomized controlled trial. The study design has been reported previously [22]. Briefly, participants were randomly assigned to administration of

Table 1 Baseline clinical characteristics by incident

\begin{tabular}{|c|c|c|c|}
\hline Characteristic & $\begin{array}{l}\text { Developed acute respiratory distress } \\
\text { syndrome (Berlin definition) } \\
(n=52)\end{array}$ & $\begin{array}{l}\text { Did not develop acute respiratory distress } \\
\text { syndrome (Berlin definition) } \\
(n=148)\end{array}$ & $P$ value \\
\hline Age (years), median (IQR) & $29.0(19.5)$ & $30.0(23.0)$ & 0.87 \\
\hline Male sex, $n(\%)$ & $50(96.2)$ & $123(83.1)$ & 0.03 \\
\hline \multicolumn{4}{|l|}{ Race, $n(\%)$} \\
\hline black & $8(15.4)$ & $35(23.6)$ & \multirow[t]{4}{*}{0.44} \\
\hline white & $16(30.8)$ & $32(21.6)$ & \\
\hline Hispanic & $27(51.9)$ & $76(51.4)$ & \\
\hline Asian & $1(1.9)$ & $5(3.4)$ & \\
\hline \multicolumn{4}{|l|}{ Emergency room sum Glasgow Coma Scale } \\
\hline$>8$ & $7(13.5)$ & $38(25.7)$ & \multirow[t]{3}{*}{0.04} \\
\hline $6-8$ & $21(40.4)$ & $68(45.9)$ & \\
\hline $3-5$ & $24(46.2)$ & $42(28.4)$ & \\
\hline $\begin{array}{l}\text { Emergency room sum Glasgow Coma Scale, } \\
\text { median (IQR) }\end{array}$ & $6.0(5.0)$ & $7.0(4.0)$ & 0.02 \\
\hline \multicolumn{4}{|l|}{ Marshall classification on computed tomography } \\
\hline mild diffuse injury I-II & $23(44.2)$ & $66(44.6)$ & \multirow[t]{3}{*}{0.22} \\
\hline severe diffuse injury III-IV & $16(30.8)$ & $30(20.3)$ & \\
\hline mass lesion & $13(25.0)$ & $52(35.1)$ & \\
\hline $\begin{array}{l}\text { Acute Physiology and Chronic Health } \\
\text { Evaluation II score, median (IQR) }\end{array}$ & $24.0(11.0)$ & $19.0(7.0)$ & $<0.001$ \\
\hline Abbreviated Injury Severity Score, median (IQR) & $32.0(14.5)$ & $28.0(10.0)$ & 0.01 \\
\hline Intracranial pressure $(n=197)$, median (IQR) & $14.0(12.0)$ & $14.0(12.0)$ & 0.84 \\
\hline \multicolumn{4}{|l|}{ Mechanism of injury, $n$ (\%) } \\
\hline assault & $7(13.5)$ & $15(10.1)$ & \multirow[t]{5}{*}{0.93} \\
\hline fall/jump & $7(13.5)$ & $20(13.5)$ & \\
\hline motor vehicle & $28(53.8)$ & $88(59.5)$ & \\
\hline Motorcycle & $9(17.3)$ & $22(14.9)$ & \\
\hline Other & $1(1.9)$ & $3(2.0)$ & \\
\hline Surgery on admission, $n(\%)$ & $14(26.9)$ & $57(38.5)$ & 0.18 \\
\hline Pre-hospital hypotension, $n$ (\%) & $7(13.5)$ & $18(12.2)$ & 0.81 \\
\hline Pre-hospital hypoxia & $16(30.8)$ & $23(15.5)$ & 0.02 \\
\hline Time (days) from injury to ALI, median (IQR) & $3.0(3.0)$ & & \\
\hline
\end{tabular}


erythropoietin or placebo and to hemoglobin transfusion thresholds of 7 or $10 \mathrm{~g} / \mathrm{dl}$ in a $2 \times 2$ factorial design. Participants with a closed head injury who were not able to follow commands after resuscitation and could be enrolled within 6 hours of injury were recruited from two level-1 trauma centers. Patients were excluded if their Glasgow Coma Scale (GCS) score was 3, and they had fixed and dilated pupils, penetrating trauma, pregnancy, life-threatening systemic injuries, or severe preexisting disease. Transfusion of leuko-reduced packed red blood cells was used to maintain the assigned hemoglobin threshold. Patients were enrolled using the emergency consent exception when relatives were not available for prospective consent. The study was approved by the local Institutional Review Boards (IRBs) at participating institutions.

\section{Cytokine and nitric oxide (NOx) measurements}

Heparinized blood samples were collected, usually via a radial artery catheter. The samples were centrifuged, aliquoted, and temporarily stored at $-80{ }^{\circ} \mathrm{C}$ in an area adjacent to the ICUs at the hospitals until they were transferred to our laboratory at Baylor College of Medicine for analysis. Both enrolling hospitals were adjacent to Baylor and the samples were transferred on dry ice from the freezer at the hospital to the freezer at Baylor where they were kept until they were analyzed.

Cytokine measurements were performed using multiplex flow cytometric bead array analysis (Beckton Dickinson Cytometric Bead Array, Human Inflammation Kit, San Jose, CA, USA) in the Cytometry and Cell Sorting
Core at Baylor College of Medicine. This method permits the simultaneous measurement of six cytokines (IL-1 $\beta$, IL6 , IL-8, IL-12, TNF $\alpha$, and IL-10) in a single sample. In this study, we evaluated IL-1 $\beta$, IL-6, IL-8, IL-12, TNF $\alpha$, IL-10 and nitric oxide (NOx). The final products of $\mathrm{NO}$ oxidation (nitrate $\left(\mathrm{NO}_{3^{-}}\right)$and nitrite $\left(\mathrm{NO}_{2}^{-}\right)$) were analyzed in plasma samples that were ultra-filtered through a $30-\mathrm{kDa}$ molecular weight cutoff filter (Millipore Corporation, Billeria, MA, USA), and then transferred to a plate to be analyzed using a Nitrate/Nitrite Fluorometric assay kit (Cayman Chemical's Company, Ann Harbor, MI, USA) using an excitation wavelength of 360-365 $\mathrm{nm}$ and an emission wavelength of $430 \mathrm{~nm}$ (Synergy 2 Multi-Mode Microplate Reader Biotek Instruments, Inc).

\section{Data analysis}

Statistical analyses were performed using SAS version 9.3 (SAS Institute Inc) and R version 2.13.1 (R Foundation for Statistical Computing) with two-sided statistical tests at a 0.05 significance level. Baseline covariates considered were transfusion threshold randomization group $(<10 \mathrm{~g} / \mathrm{dl} ;<7 \mathrm{~g} /$ $\mathrm{dl}$ ); erythropoietin (EPO) randomization group (Epo1 or Epo2 if randomized to the first or second erythropoietin dosing regimens, respectively, versus placebo); pre-hospital hypotension present; pre-hospital hypoxia present; age (years); emergency room (ER) GCS-sum; Injury Severity Score (ISS); number of unreactive pupils (one or two versus none); ER computed tomography (CT) high risk; and intubated in ER (versus ICU or in the field) at baseline. The first measurement of cytokines within 24 hours after injury was
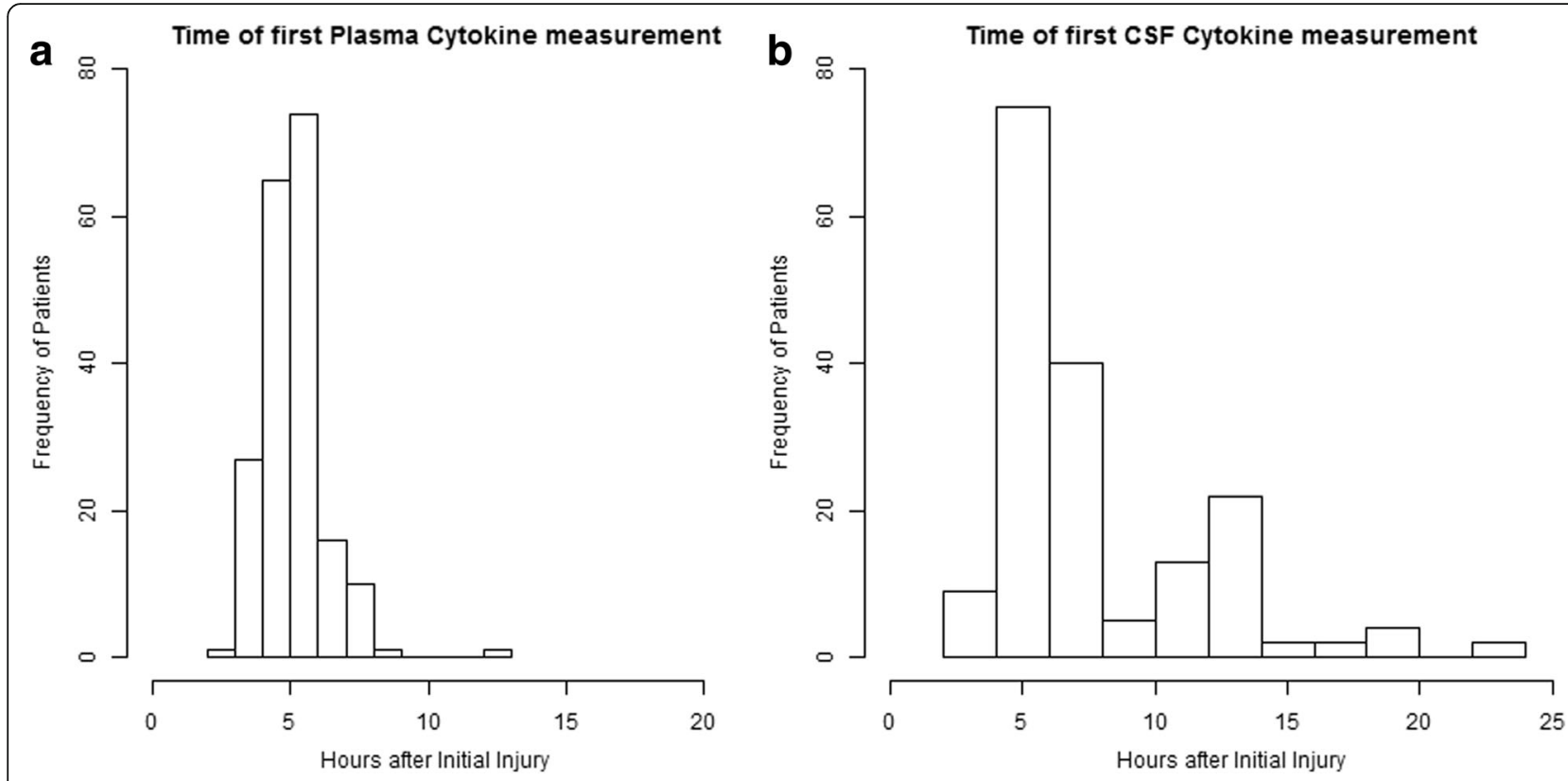

Fig. 1 a Distribution of time of plasma cytokine measurement after injury. $\mathbf{b}$ Distribution of time of cerebrospinal fluid cytokine measurement after injury 
Table 2 Comparison of plasma cytokines among patients who had or did not have ARDS

\begin{tabular}{|c|c|c|c|c|}
\hline Cytokine & Normal ranges (pg/mL) & $\begin{array}{l}\text { ARDS median }\left(25^{\text {th }}, 75^{\text {th }}\right)(\text { min-max }) \\
(n=51)\end{array}$ & $\begin{array}{l}\text { No ARDS median }\left(25^{\text {th }}, 75^{\text {th }}\right)(\text { min-max }) \\
(n=144)\end{array}$ & $P$ value \\
\hline IL-12p70 $(n=194)$ & $0-6$ & $3.9(2.2,5.9)(0.1-163.6)$ & $3.8(2.7,5.4)(0.2-40.7)$ & 0.882 \\
\hline IL-10 $(n=195)$ & $0-18$ & $14.5(8.1,34.8)(0.2-147.6)$ & $10.0(6.1,18.6)(0.2-98.2)$ & 0.008 \\
\hline IL-8 $(n=194)$ & $0-5$ & $158.1(45.0,284.0)(0.04-2504.0)$ & $57.4(26.0,135.1)(0.1-7393.0)$ & $<0.001$ \\
\hline IL-6 $(n=195)$ & $0-5$ & $568.2(224.4,2278.0)(5.9-18,950)$ & $241.2(92.7,638.8)(3.0-21,330.0)$ & $<0.001$ \\
\hline $\operatorname{IL}-1 \beta(n=195)$ & $0-39$ & $5.4(3.1,9.0)(0.1-54)$ & $4.7(3.2,8.3)(0.1-142.8)$ & 0.702 \\
\hline Nitric oxide $(n=193)$ & Not available & $1.7(1.3,2.5)(0.01-14.8)$ & $1.5(1.0,2.4)(0.01-7.3)$ & 0.266 \\
\hline TNF-a $(n=195)$ & $0-22$ & $3.8(1.8,6.2)(0.1-38.8)$ & $3.9(3.2,5.4)(0.3-95.9)$ & 0.743 \\
\hline
\end{tabular}

used as the baseline value. Although some measurements were not obtained before transfusion or before EPO administration, there was no difference in ALI/ARDS between the intervention and control arms of the randomized trial [22].

The Wilcoxon rank sum test or Fisher's exact test was used to compare continuous and categorical variables, respectively, among patients who did or did not develop ARDS. Cox proportional hazards regression was used to determine whether the cytokine values were associated with an increased risk of ARDS, adjusted for baseline variables. Due to the large number of potential baseline variables and the relatively small number of ARDS events, variables were selected to build a parsimonious model. Lasso-penalized Cox [23] regression, with the penalty parameter selected using fivefold cross-validation, was used for selection of variables in addition to the transfusion threshold randomization group and all baseline variables that were significantly different among patients who did or did not have an ARDS event. The lasso penalization has better statistical properties than other common methods for variable selection, such as forward or backward stepwise selection, which tend to result in underestimation of the standard errors of the coefficients. Censor time was defined as 6 months after injury, date of hospital discharge, death, or withdrawal, whichever occurred first. Spearman's rank correlation (rho) was used to assess the correlation between the initial cytokine value and the category of ARDS severity based on the Berlin definition of ARDS.

\section{Results}

A total of 200 patients were enrolled in the study of whom between 173 and 195 (87-98 \%) were included in the cytokine analyses, as there were varying numbers of missing values for each cytokine. Patients were excluded if they did not have any cytokine values available within 24 hours of injury or if they had an ARDS event on the first day, because we did not know whether the cytokine measurement was obtained before or after the ARDS event. The baseline clinical characteristics are presented in Table 1 . There were some significant differences in some baseline characteristics between patients with and without ARDS, notably a significant difference in gender $(96.2 \%$ male $(50 / 52)$ vs. $83.1 \%$ $(123 / 148)$, respectively, $p=0.03)$. The GCS sum score in the ER (median $=6$ vs. 7, $p=0.02$ ), Acute Physiology and Chronic Health Evaluation (APACHE) II score (24 vs. 19, $p$ $<0.001$ ), Abbreviated Injury Severity Score (median $=32$ vs. $28, p=0.01$ ), and pre-hospital hypoxia (present in $30.8 \%$ of patients $(16 / 52)$ vs. $15.5 \%(23 / 148))$, respectively. The majority (86\%) of plasma cytokines were collected within 6 hours of injury (Fig. 1a) and $48 \%$ of CSF cytokines were collected within 6 hours of injury (Fig. 1b).

Table 3 Comparison of cerebrospinal fluid cytokines among patients who did or did not have acute respiratory distress syndrome (ARDS) and those who did not

\begin{tabular}{lllll}
\hline Cytokine & Normal ranges $(\mathrm{pg} / \mathrm{mL})$ & $\begin{array}{l}\text { ARDS median }\left(25^{\text {th }}, 75^{\text {th }}\right)(\mathrm{min}-\mathrm{max}) \\
(n=45)\end{array}$ & $\begin{array}{l}\text { No ARDS median }\left(25^{\text {th }}, 75^{\text {th }}\right)(\mathrm{min}-\mathrm{max}) \\
(n=129)\end{array}$ \\
\hline IL-12p70 $(n=173)$ & $0-2$ & $3.8(2.3,5.7)(0.1-54.4)$ & $3.5(0.9,4.1)(0.1-45.9)$ & 0.155 \\
IL-10 $(n=174)$ & $0-1.5$ & $7.0(4.3,15.6)(0.2-78.4)$ & $5.1(3.5,10.0)(0.2-303.7)$ & 0.026 \\
IL-8 $(n=173)$ & $0-2$ & $1203.0(93.7,4782.0)(3.9-32,570.0)$ & $985.9(72.6,6792.0)(0.04-204,900)$ & 0.716 \\
IL-6 $(n=174)$ & $0-1$ & $1820.0(233.3,7083.0)(0.2-17,790.0)$ & $609.4(58.6,2917.0)(0.2-76,370)$ & 0.085 \\
IL-1 $(n=174)$ & $0-3$ & $5.6(3.7,8.0)(0.1-74.6)$ & $5.2(3.5,8.1)(0.1-82.4)$ & 0.490 \\
Nitric oxide $(n=172)$ & Not available & $0.5(0.3,0.9)(0.01-3.6)$ & $0.5(0.1,0.7)(0.01-3.98)$ & 0.198 \\
TNF-a $(n=174)$ & $0-2$ & $4.2(2.1,6.3)(0.3-38.8)$ & $3.8(2.0,4.3)(0.3-30.3)$ & 0.179 \\
\hline
\end{tabular}


Table 4 Associations between each plasma cytokine and time to development of acute respiratory distress syndrome from Cox regression analysis in erythropoietin study subjects with traumatic brain injury (unadjusted and adjusted analyses)

\begin{tabular}{|c|c|c|c|c|c|c|c|}
\hline & & Unac & & & Adjus & & \\
\hline & Number & $\mathrm{HR}$ & $95 \% \mathrm{Cl}$ & $P$ value & $\mathrm{HR}$ & $95 \% \mathrm{Cl}$ & $P$ value \\
\hline IL-12p70 & 194 & 1.03 & $0.74,0.14$ & 0.88 & 1.18 & $0.82,1.69$ & 0.36 \\
\hline Tा10 & & & & & 1.24 & $0.70,2.19$ & 0.46 \\
\hline ER sum GCS & & & & & 0.86 & $0.76,0.98$ & 0.02 \\
\hline Pre-hospital hypoxia & & & & & 1.71 & $0.88,3.33$ & 0.11 \\
\hline Male sex & & & & & 4.97 & $1.17,21.16$ & 0.03 \\
\hline ISS & & & & & 1.04 & $1.00,1.07$ & 0.03 \\
\hline Intubated in ER & & & & & 1.51 & $0.72,3.17$ & 0.28 \\
\hline Pre-hospital hypotension & & & & & 0.62 & $025,1.56$ & 0.31 \\
\hline Pupil unreactive (1-2 vs. 0) & & & & & 0.65 & $0.35,1.22$ & 0.18 \\
\hline TNF-a & 195 & 0.96 & $0.67,1.37$ & 0.81 & 1.03 & $0.71,1.51$ & 0.85 \\
\hline Tा10 & & & & & 1.17 & $0.067,2.07$ & 0.56 \\
\hline ISS & & & & & 1.03 & $0.99,1.07$ & 0.05 \\
\hline Male sex & & & & & 4.79 & $1.15,20.06$ & 0.03 \\
\hline ER sum GCS & & & & & 0.86 & $0.76,0.98$ & 0.02 \\
\hline Pre-hospital hypoxia & & & & & 1.78 & $0.92,3.43$ & 0.08 \\
\hline Pre-hospital hypotension & & & & & 0.62 & $025,1.54$ & 0.30 \\
\hline Pupil unreactive (1-2 vs. 0) & & & & & 0.67 & $0.36,1.25$ & 0.21 \\
\hline Intubated in ER & & & & & 1.55 & $0.74,3.25$ & 0.25 \\
\hline IL-10 & 195 & 1.57 & $1.17,2.11$ & $<0.01$ & 1.66 & $1.22,2.26$ & $<0.01$ \\
\hline Tा10 & & & & & 1.21 & $0.69,2.11$ & 0.51 \\
\hline ER sum GCS & & & & & 0.86 & $0.76,0.98$ & 0.02 \\
\hline Pre-hospital hypoxia & & & & & 2.00 & $0.98,3.73$ & 0.06 \\
\hline Male sex & & & & & 5.23 & $1.23,22.29$ & 0.03 \\
\hline ISS & & & & & 1.03 & $0.99,1.06$ & 0.11 \\
\hline Pre-hospital hypotension & & & & & 0.50 & $0.20,1.23$ & 0.13 \\
\hline Pupil unreactive (1-2 vs. 0) & & & & & 0.56 & $0.30,1.05$ & 0.07 \\
\hline IL-6 & 195 & 1.30 & $1.11,1.52$ & $<0.01$ & 1.24 & $1.05,1.49$ & 0.01 \\
\hline$\pi 10$ & & & & & 1.14 & $0.65,1.99$ & 0.65 \\
\hline Pre-hospital hypoxia & & & & & 1.64 & $0.85,3.16$ & 0.14 \\
\hline Male sex & & & & & 4.75 & $1.13,19.95$ & 0.03 \\
\hline ER sum GCS & & & & & 0.89 & $0.79,1.01$ & 0.06 \\
\hline ISS & & & & & 1.02 & $0.99,1.06$ & 0.22 \\
\hline Intubated in ER & & & & & 1.50 & $0.71,3.18$ & 0.29 \\
\hline Pre-hospital hypotension & & & & & 0.52 & $0.21,1.28$ & 0.15 \\
\hline Pupil unreactive (1-2 vs. 0) & & & & & 0.60 & $0.32,1.12$ & 0.11 \\
\hline $\mid L-1 \beta$ & 195 & 0.98 & $0.73,1.30$ & 0.87 & 0.98 & $0.73,1.32$ & 0.89 \\
\hline TT10 & & & & & 1.17 & $0.67,2.05$ & 0.58 \\
\hline ISS & & & & & 1.03 & $1.00,1.07$ & 0.06 \\
\hline ER sum GCS & & & & & 0.86 & $0.76,0.98$ & 0.02 \\
\hline Pre-hospital hypoxia & & & & & 0.62 & $0.25,1.55$ & 0.09 \\
\hline Pupil unreactive (1-2 vs. 0) & & & & & 0.67 & $0.36,1.25$ & 0.21 \\
\hline Male sex & & & & & 4.75 & $1.13,20.0$ & 0.03 \\
\hline
\end{tabular}


Table 4 Associations between each plasma cytokine and time to development of acute respiratory distress syndrome from Cox regression analysis in erythropoietin study subjects with traumatic brain injury (unadjusted and adjusted analyses) (Continued)

\begin{tabular}{|c|c|c|c|c|c|c|c|}
\hline Intubated in ER & & & & & 1.57 & $0.74,3.30$ & 0.24 \\
\hline Pre-hospital hypotension & & & & & 0.62 & $0.25,1.55$ & 0.31 \\
\hline IL-8 & 194 & 1.33 & $1.11,1.59$ & $<0.01$ & 1.26 & $1.04,1.53$ & 0.02 \\
\hline Tा10 & & & & & 1.04 & $0.58,1.87$ & 0.89 \\
\hline ISS & & & & & 1.02 & $0.98,10.5$ & 0.62 \\
\hline ER sum GCS & & & & & 0.90 & $0.80,10.2$ & 0.10 \\
\hline Pre-hospital hypoxia & & & & & 1.94 & $1.01,3.74$ & 0.05 \\
\hline Pupil unreactive (1-2 vs. 0 ) & & & & & 0.61 & $0.33,1.15$ & 0.12 \\
\hline Male Sex & & & & & 4.20 & $1.01,17.53$ & 0.05 \\
\hline Pre-hospital hypotension & & & & & 0.55 & $0.22,1.35$ & 0.19 \\
\hline NOx & 193 & 1.64 & $0.90,3.00$ & 0.11 & 1.60 & $0.89,2.90$ & 0.12 \\
\hline TT10 & & & & & 1.12 & $0.64,1.97$ & 0.69 \\
\hline ISS & & & & & 1.03 & $1.00,1.06$ & 0.06 \\
\hline ER sum GCS & & & & & 0.91 & $0.81,1.03$ & 0.13 \\
\hline Pre-hospital hypotension & & & & & 0.56 & $0.22,1.41$ & 0.22 \\
\hline Pre-hospital hypotension & & & & & 1.84 & $0.95,3.56$ & 0.07 \\
\hline Male sex & & & & & 4.73 & $1.14,19.62$ & 0.03 \\
\hline
\end{tabular}

Variables considered for adjustment included age, injury severity score (ISS), emergency room (ER) sum Glasgow Coma Scale (GCS), unreactive pupils, emergency room computer tomography indicating high risk, pre-hospital hypotension, pre-hospital hypoxia, intubated in the ER, transfusion threshold, and each baseline cytokine level. Cytokine level, transfusion threshold, pre-hospital hypoxia, ER sum GCS, sex, and ISS were forced into the adjusted model. HR hazard ratio, IL-12p70 interleukin-12 p70, TNF- $a$ tumor necrosis factor alpha, IL-10 interleukin-10, IL-6 interleukin-6, IL-1 $\beta$ interleukin- 1 beta, $I L-8$ interleukin-8, NOx nitric oxide, $T 110$ transfusion threshold $<10 \mathrm{~g} / \mathrm{dL}$

Plasma and CSF cytokine levels by ARDS status are presented in Tables 2 and 3. Median IL-6, IL-8, and IL$10(\mathrm{IL}-6=568.2, \mathrm{IL}-8=158.1$, and $\mathrm{IL}-10=14.5 \mathrm{pg} / \mathrm{mL})$ in plasma from the ARDS group was significantly higher than in the non-ARDS group (IL-6 $=241.2$, IL_ $8=57.4$, and $\mathrm{IL}-10=10.0 \mathrm{pg} / \mathrm{mL}$ ). In CSF, median IL-10 was 7.0 and $5.1 \mathrm{pg} / \mathrm{mL}$ in patients with and without ARDS, respectively, with a $p$ value of 0.026 .

Cox proportional hazard ratios (HR) for time to ARDS are shown in Tables 4 and 5 . We observed $66 \%$ higher risk for each unit increase in plasma IL-10 (HR $=1.66,95 \% \mathrm{CI}$ $=1.22,2.26, p=0.001)$. Plasma IL-6 and IL-8 were associated with $24 \%$ and $26 \%$ higher risk, respectively, for each unit increase in cytokine value (IL-6 adjusted HR $=1.24$, $95 \% \mathrm{CI}=1.05$. 1.49, $p=0.012$; IL-8 adjusted $\mathrm{HR}=1.26$, $95 \% \mathrm{CI}=1.04,1.49, p=0.02)$. CSF IL-12 was associated with $52 \%$ increased risk for each unit increase (adjusted $\mathrm{HR}=1.52,95 \% \mathrm{CI}=1.05,2.21, p=0.03$ ). CSF IL-10 was associated with $33 \%$ increased risk for each unit increase and was statistically significant (adjusted $\mathrm{HR}=1.33,95 \% \mathrm{CI}=$ $1.00,1.76, p=0.05)$. There were no significant differences in other proinflammatory and anti-inflammatory markers in either the plasma or CSF (Tables 2, 3, 4 and 5). There was positive correlation between the initial plasma cytokine value and the Berlin definition of ARDS by severity: for IL6 rho $=0.12, p<0.001$, for IL-8 rho $=0.14, p<0.001$, and for IL-10 rho $=0.15, p<0.001$.
High tidal volume ventilator strategies are known to induce volume trauma and barotrauma and low tidal volume strategies may be protective in patients with ARDS. The median initial tidal volume, i.e., prior to the development of ARDS, was $8.52 \mathrm{~mL} / \mathrm{kg}\left(25^{\text {th }}-75^{\text {th }}\right.$ percentiles $=8.18-9.10)$ for patients who developed ARDS $(n=35)$ and $9.02 \mathrm{~mL} / \mathrm{kg}\left(25^{\text {th }}-75^{\text {th }}\right.$ percentiles $=8.38-$ 9.76) for patients who did not develop ARDS $(n=93)$, and this was statistically significantly different $(p=0.04)$.

\section{Discussion}

Acute respiratory distress syndrome is a well-described complication in patients who survive an initial traumatic insult. Inflammatory responses to TBI, both local and systemic, are complex. Invading cells from the blood (e.g., leukocytes, monocytes and macrophages) and/or activated resident cells (e.g., microglia) release a number of inflammatory molecules and free radicals that can contribute to brain edema and worsen neurological outcome. Increased serum and/or CSF concentrations of proinflammatory and anti-inflammatory cytokines, chemokines, and acute phase reactant proteins have been observed following TBI. The importance of inflammation in the progression of TBIassociated pathologic change has been the focus of numerous studies [1, 24, 25]. More recently, the expression of these factors has been appreciated as a potential marker 
Table 5 Associations between each cytokine measured in cerebrospinal fluid and time to development of acute respiratory distress syndrome from Cox regression analysis in erythropoietin study subjects with traumatic brain injury (unadjusted and adjusted analyses)

\begin{tabular}{|c|c|c|c|c|c|c|c|}
\hline & & Unac & & & Adjus & & \\
\hline & Number & $\overline{\mathrm{HR}}$ & $95 \% \mathrm{Cl}$ & $P$ value & $\overline{\mathrm{HR}}$ & $95 \% \mathrm{Cl}$ & $P$ value \\
\hline IL-12p70 & 173 & 1.36 & $0.94,1.95$ & 0.10 & 1.52 & $1.04,2.21$ & 0.03 \\
\hline TT10 & & & & & 1.12 & $0.62,2.04$ & 0.69 \\
\hline Pre-hospital hypoxia & & & & & 1.38 & $0.68,2.79$ & 0.38 \\
\hline Male sex & & & & & 8.69 & $1.17,64.53$ & 0.03 \\
\hline ER sum GCS & & & & & 0.90 & $0.79,1.03$ & 0.11 \\
\hline ISS & & & & & 1.04 & $1.00,1.07$ & 0.04 \\
\hline TNF-a & 174 & 1.22 & $0.83,1.80$ & 0.32 & 1.43 & $0.97,2.14$ & 0.08 \\
\hline TT10 & & & & & 1.08 & $0.60,1.95$ & 0.80 \\
\hline Pre-hospital hypoxia & & & & & 1.27 & $0.63,2.58$ & 0.51 \\
\hline Male sex & & & & & 10.40 & $1.38,78.61$ & 0.02 \\
\hline ER sum GCS & & & & & 0.86 & $0.75,0.99$ & 0.03 \\
\hline ISS & & & & & 1.04 & $1.01,1.08$ & 0.02 \\
\hline$\| \mathrm{L}-10$ & 174 & 1.27 & $0.99,1.64$ & 0.06 & 1.33 & $1.00,1.76$ & 0.05 \\
\hline TT10 & & & & & 1.27 & $0.69,2.35$ & 0.43 \\
\hline Pre-hospital hypoxia & & & & & 1.57 & $0.64,0.79$ & 0.20 \\
\hline Male sex & & & & & 6.98 & $0.95,51.31$ & 0.06 \\
\hline ER sum GCS & & & & & 0.87 & $0.76,0.99$ & 0.04 \\
\hline ISS & & & & & 1.02 & $0.99,1.05$ & 0.18 \\
\hline $\mathrm{IL}-.6$ & 174 & 1.09 & $0.97,1.21$ & 0.14 & 1.06 & $0.95,1.19$ & 0.28 \\
\hline TT10 & & & & & 1.06 & $0.59,1.92$ & 0.83 \\
\hline Pre-hospital hypoxia & & & & & 1.41 & $0.70,2.82$ & 0.33 \\
\hline Male sex & & & & & 7.60 & $1.04,55.59$ & $0.05^{*}$ \\
\hline ER sum GCS & & & & & 0.91 & $0.81,1.03$ & 0.15 \\
\hline ISS & & & & & 1.02 & $0.99,1.06$ & 0.14 \\
\hline$I L-1 \beta$ & 174 & 1.05 & $0.77,1.43$ & 0.76 & 1.11 & $0.80,1.54$ & 0.54 \\
\hline T110 & & & & & 0.91 & $0.60,2.01$ & 0.76 \\
\hline Pre-hospital hypoxia & & & & & 1.30 & $0.64,2.65$ & 0.47 \\
\hline Male sex & & & & & 9.25 & $1.24,69.18$ & 0.03 \\
\hline ER sum GCS & & & & & 0.88 & $0.78,1.01$ & 0.06 \\
\hline ISS & & & & & 1.04 & $1.00,1.08$ & 0.14 \\
\hline $\mid \mathrm{L}-8$ & 173 & 1.02 & $0.92,1.13$ & 0.70 & 1.01 & $0.92,1.12$ & 0.81 \\
\hline Tा10 & & & & & 1.11 & $0.61,2.01$ & 0.73 \\
\hline Pre-hospital hypoxia & & & & & 1.42 & $0.70,2.88$ & 0.32 \\
\hline Male sex & & & & & 7.68 & $1.05,56.22$ & 0.04 \\
\hline ER sum GCS & & & & & 0.92 & $0.81,1.04$ & 0.19 \\
\hline ISS & & & & & 1.03 & $0.99,1.06$ & 0.11 \\
\hline NOx & 172 & 2.11 & $0.89,0.502$ & .09 & 1.66 & $0.70,3.97$ & 0.25 \\
\hline T10 & & & & & 1.17 & $0.64,2.13$ & 0.61 \\
\hline Pre-hospital hypoxia & & & & & 1.39 & $0.67,2.88$ & 0.37 \\
\hline
\end{tabular}


Table 5 Associations between each cytokine measured in cerebrospinal fluid and time to development of acute respiratory distress syndrome from Cox regression analysis in erythropoietin study subjects with traumatic brain injury (unadjusted and adjusted analyses) (Continued)

\begin{tabular}{llll}
\hline Male sex & 6.76 & $0.91,49.9$ & 0.06 \\
ER sum GCS & 0.89 & $0.78,1.02$ & 0.09 \\
ISS & 1.02 & $0.99,1.06$ & 0.14
\end{tabular}

Variables considered for adjustment included age, injury severity score (ISS), enrollment sum Glasgow Coma Scale (GCS), unreactive pupils, emergency room (ER) computed tomography indicating high risk, pre-hospital hypotension, pre-hospital hypoxia, intubated in the ER, transfusion threshold, and each baseline cytokine level. Cytokine level, transfusion threshold, pre-hospital hypoxia, ER sum GCS, sex, and ISS were forced into the adjusted model. $H R$ hazard ratio, IL-12p70 interleukin-12 p70, TNF- $a$ tumor necrosis factor alpha, IL-10 interleukin-10, IL-6 interleukin-6, IL-1 $\beta$ interleukin- 1 beta, IL-8 interleukin-8, NOx nitric oxide, $T 110$ transfusion threshold $<10 \mathrm{~g} / \mathrm{dL}$

of injury, and studies have been carried out to test whether these factors correlate with outcome or can serve as surrogate markers of treatment efficacy.

TBI biomarkers have primarily focused on prediction of elevated intracranial pressure or mortality. Several biomarkers have emerged as possible markers of significant neurological injury, including s100B, IL-8, and ceruloplasmin. There are no data on biomarkers that predict ARDS in patients with severe TBI, despite incidence of 20-30\% and high mortality. In two separate studies in the late 1990s [16, 17], Parsons and Pittet did not identify any association with inflammatory cytokines in non-TBI in atrisk patients with ARDS.

Our study is the first to identify correlation between initial cytokines in patients with TBI and the development of ARDS. Elevated plasma markers of proinflammatory cytokines IL- 6 and IL-8 and anti-inflammatory marker IL-10 may indicate the importance of an extracranial inflammatory response in the development of ARDS. CSF cytokines IL-12 and IL-10 were statistically significant in a fully adjusted model for markers of severity of illness, which we report in Table 5. CSF IL-12 and IL-10 are potentially correlated with ARDS in patients with TBI, but is difficult to confidently interpret this result and we suggest the results be cautiously interpreted and that additional studies may be required to confirm these associations. Severe TBI likely induces an inflammatory response that is not limited to the brain and the TBI-mediated systemic response is likely involved in the development of ARDS, and may be quantifiable in the plasma. This is particularly relevant clinically and in research, as early plasma markers are easier to obtain than CSF.

The majority of cytokine levels were drawn within 6 hours of injury. The time frame within which the cytokines were measured suggests an inflammatory response that is triggered from the initial intracranial injury. Interestingly, elevated ICP, a known prognosticator of mortality and a marker of disease severity in patients with severe TBI [26], and the Marshall CT classification of injury were similar in the ARDS and non ARDS groups and therefore did not impact the development of ARDS.
It is worth noting that other potential triggers of an inflammatory response were considered, including mechanical ventilation and transfusion. High tidal volume mechanical ventilation is known to be deleterious and contribute to ventilator-induced lung injury (VILI) [27, 28]. Patients with severe TBI frequently have elevated ICP, which makes low tidal volume lung-protective ventilation [29] and permissive hypercapnea challenging strategies to employ. The tidal volume in our study population was modest and below typical thresholds for high tidal-volume ventilation. Ventilator strategy likely did not contribute to the incidence of ARDS as there was no difference between the two groups. Critical to the design of the study was the randomization of patients to prespecified transfusion triggers. The baseline cytokine values reflect the period before administration of any blood products, and we have previously reported [22] that there was no difference in the incidence of ARDS between the intervention and control groups. Physicians caring for patients with severe TBI should be aware of the complex neuroinflammatory mechanisms that are associated with ARDS in patients with TBI and should consider strategies to minimize lung injury early in the treatment algorithm in patients who survive the initial injury.

Our study has several limitations worth acknowledging. The study is a retrospective and secondary analysis of prospectively collected data on erythropoietin in severe TBI. However, we feel this does not impact the results or strength of the study as the variables and outcomes were selected and collected prospectively. The cytokine measurements were single as opposed to sequential measurements. While sequential measurements may provide additional information, we felt sequential measurements would be more difficult to interpret and could potentially be influenced by our primary interventional study. Therefore, initial cytokine concentrations were used for analysis. Our study protocol did not require lung-protective strategies in all patients. However, there was no statistical difference in mean tidal volume between our groups and we do not believe this contributed to the development of ARDS. 


\section{Conclusion}

Elevated plasma inflammatory markers IL-6, IL-8, and IL10 in the early phase of severe acute TBI are associated with the development of ARDS. Additional studies to validate these findings are needed.

\section{Abbreviations}

ALI: acute lung injury; APACHE: Acute Physiology and Chronic Health Evaluation; ARDS: acute respiratory distress syndrome; CSF: cerebrospinal fluid; CT: computed tomography; EPO: erythropoietin; ER: emergency room; GCS: Glasgow Coma Scale; HR: hazard ratio; ICP: intracranial pressure; ICU: Intensive Care Unit; IRB: Institutional Review Board; ISS: Injury Severity Scale; SIRS: systemic inflammatory response syndrome; TBI: traumatic brain injury; VILI: ventilator-induced lung injury

\section{Acknowledgements}

This study was supported by the National Institute of Neurological Disorders and Stroke (grant \#P01-NS38660), the Cytometry and Cell Sorting Core at Baylor College of Medicine with funding from the NIH (Al036211, CA125123, and RR024574), and the expert assistance of Joel M. Sederstrom.

\section{Funding}

NIH Grant: P01-NS38660

\section{Availability of data and materials}

The datasets used and/or analyzed during the current study are available from the study principal investigator or corresponding author on reasonable request.

\section{Authors' contributions}

Author contributions are consistent with ICMJE recommendations. All authors are in agreement to be accountable for all aspects of the work in ensuring that questions related to the accuracy or integrity of any part of the work are appropriately investigated and resolved. IA contributed to the drafting of the manuscript, data acquisition, patient recruitment, data interpretation, and overall study participation. JY contributed to the data analysis, interpretation, and drafting/revisions of the manuscript. PD contributed to the patient recruitment, interpretation, and drafting/revisions of the manuscript. JS contributed to the data analysis, interpretation, and drafting/revisions of the manuscript. SG contributed to the patient recruitment and drafting/revisions of the manuscript. JG contributed by carrying out the cytokine measurements and drafting/revisions of the manuscript. CR contributed to study design, patient recruitment, data acquisition, data analysis, interpretation, and drafting/revisions of the manuscript. All authors read and approved the final manuscript.

\section{Authors' information}

Not applicable.

\section{Competing interests}

The authors declare that they have no competing interests.

\section{Consent for publication}

Not applicable.

\section{Ethics approval and consent to participate}

Ethical approval and consent was obtained from the local IRBs to participate. The study was approved under waiver of consent. These included the IRBS from University of Texas Houston Medical School, Baylor College of Medicine, Memorial Hermann Hospital, and BenTaub Hospital.

\section{Author details}

'Department of Emergency Medicine, Brigham and Women's Hospital, 75 Francis Street, Boston, MA 02115, USA. 'Division of Biostatistics, University of Texas School of Public Health, Houston, TX, USA. ${ }^{3}$ Department of Emergency Medicine and Internal Medicine, University of Texas Health Science Center at Houston, Houston, TX, USA. ${ }^{4}$ Department of Basic Vision Sciences, College of Optometry Texas Institute for Measurement Evaluation and Statistics, University of Houston, Houston, TX, USA. ${ }^{5}$ Department of Neurosurgery, Baylor College of Medicine, Houston, TX, USA. 'Department of Pathology, Baylor College of Medicine, Houston, TX, USA.
Received: 24 June 2016 Accepted: 26 August 2016

Published online: 15 September 2016

\section{References}

1. Kadhim HJ, Duchateau J, Sebire G. Cytokines and brain injury: invited review. J Intensive Care Med. 2008;23(4):236-49.

2. Soriano SG, Piva S. Central nervous system inflammation. Eur J Anaesthesiol Suppl. 2008;42:154-9.

3. Engelhardt B. Immune cell entry into the central nervous system: involvement of adhesion molecules and chemokines. J Neurol Sci. 2008;274(1-2):23-6.

4. Schneider Soares FM, Menezes de Souza N, Liborio Schwarzbold M, et al. Interleukin-10 is an independent biomarker of severe traumatic brain injury prognosis. Neuroimmunomodulation. 2012;19(6):377-85.

5. Lu J, Goh SJ, Tng PY, Deng YY, Ling EA, Moochhala S. Systemic inflammatory response following acute traumatic brain injury. Front Biosci. 2009;14:3795-813.

6. Gullo Jda S, Bertotti MM, Silva CC, et al. Hospital mortality of patients with severe traumatic brain injury is associated with serum PTX3 levels. Neurocrit Care. 2011;14(2):194-9.

7. Schwarzbold ML, Rial D, De Bem T, et al. Effects of traumatic brain injury of different severities on emotional, cognitive, and oxidative stress-related parameters in mice. J Neurotrauma. 2010;27(10):1883-93.

8. D'Mello C, Le T, Swain MG. Cerebral microglia recruit monocytes into the brain in response to tumor necrosis factor alpha signaling during peripheral organ inflammation. J Neurosci. 2009:29(7):2089-102.

9. Goodman JC, Van M, Gopinath SP, Robertson CS. Pro-inflammatory and proapoptotic elements of the neuroinflammatory response are activated in traumatic brain injury. Acta Neurochir Suppl. 2008;102:437-9.

10. Hergenroeder GW, Moore AN, McCoy Jr JP, et al. Serum IL-6: a candidate biomarker for intracranial pressure elevation following isolated traumatic brain injury. J Neuroinflammation. 2010;7:19.

11. Siler TM, Swierkosz JE, Hyers TM, Fowler AA, Webster RO. Immunoreactive interleukin-1 in bronchoalveolar lavage fluid of high-risk patients and patients with the adult respiratory distress syndrome. Exp Lung Res. 1989; 15(6):881-94.

12. Schutte H, Lohmeyer J, Rosseau S, et al. Bronchoalveolar and systemic cytokine profiles in patients with ARDS, severe pneumonia and cardiogenic pulmonary oedema. Eur Respir J. 1996;9(9):1858-67.

13. Suter PM, Suter S, Girardin E, Roux-Lombard P, Grau GE, Dayer JM. High bronchoalveolar levels of tumor necrosis factor and its inhibitors, interleukin-1, interferon, and elastase, in patients with adult respiratory distress syndrome after trauma, shock, or sepsis. Am Rev Respir Dis. 1992;145(5):1016-22.

14. Park WY, Goodman RB, Steinberg KP, et al. Cytokine balance in the lungs of patients with acute respiratory distress syndrome. Am J Respir Crit Care Med. 2001;164(10 Pt 1):1896-903.

15. Donnelly SC, Strieter RM, Reid PT, et al. The association between mortality rates and decreased concentrations of interleukin-10 and interleukin-1 receptor antagonist in the lung fluids of patients with the adult respiratory distress syndrome. Ann Intern Med. 1996:125(3):191-6.

16. Pittet JF, Mackersie RC, Martin TR, Matthay MA. Biological markers of acute lung injury: prognostic and pathogenetic significance. Am J Respir Crit Care Med. 1997;155(4):1187-205.

17. Parsons PE, Moss M, Vannice JL, Moore EE, Moore FA, Repine JE. Circulating IL-1ra and IL-10 levels are increased but do not predict the development of acute respiratory distress syndrome in at-risk patients. Am J Respir Crit Care Med. 1997;155(4):1469-73.

18. Maier S, Emmanuilidis K, Entleutner M, et al. Massive chemokine transcription in acute renal failure due to polymicrobial sepsis. Shock. 2000; 14(2):187-92.

19. Donnelly SC, Strieter RM, Kunkel SL, et al. Interleukin-8 and development of adult respiratory distress syndrome in at-risk patient groups. Lancet. 1993; 341(8846):643-7.

20. Goris RJ. Mediators of multiple organ failure. Intensive Care Med. 1990;16 Suppl 3:S192-6.

21. Force ADT, Ranieri VM, Rubenfeld GD, et al. Acute respiratory distress syndrome: the Berlin definition. JAMA. 2012;307(23):2526-33.

22. Robertson CS, Hannay HJ, Yamal JM, et al. Effect of erythropoietin and transfusion threshold on neurological recovery after traumatic brain injury: a randomized clinical trial. JAMA. 2014;312(1):36-47.

23. Tibshirani R. The lasso method for variable selection in the Cox model. Stat Med. 1997;16(4):385-95. 
24. Morganti-Kossmann MC, Rancan M, Stahel PF, Kossmann T. Inflammatory response in acute traumatic brain injury: a double-edged sword. Curr Opin Crit Care. 2002;8(2):101-5.

25. Whitney NP, Eidem TM, Peng H, Huang Y, Zheng JC. Inflammation mediates varying effects in neurogenesis: relevance to the pathogenesis of brain injury and neurodegenerative disorders. J Neurochem. 2009;108(6):1343-59.

26. Ruff RM, Marshall LF, Crouch J, et al. Predictors of outcome following severe head trauma: follow-up data from the Traumatic Coma Data Bank. Brain Inj. 1993;7(2):101-11.

27. Gattinoni L, Protti A, Caironi P, Carlesso E. Ventilator-induced lung injury: the anatomical and physiological framework. Crit Care Med. 2010;38(10 Suppl): S539-48.

28. Kuchnicka K, Maciejewski D. Ventilator-associated lung injury. Anaesthesiology Interv Ther. 2013;45(3):164-70.

29. The Acute Respiratory Distress Syndrome Network. Ventilation with lower tidal volumes as compared with traditional tidal volumes for acute lung injury and the acute respiratory distress syndrome. N Engl J Med. 2000;342(18):1301-8.

Submit your next manuscript to BioMed Central and we will help you at every step:

- We accept pre-submission inquiries

- Our selector tool helps you to find the most relevant journal

- We provide round the clock customer support

- Convenient online submission

- Thorough peer review

- Inclusion in PubMed and all major indexing services

- Maximum visibility for your research

Submit your manuscript at www.biomedcentral.com/submit 